\title{
Adoptive cell therapy with autologous tumor-infiltrating lymphocytes and high-dose interleukin-2 for metastatic melanoma: The surgeon's perspective
}

\author{
DOUGLAS B. ZIPPEL ${ }^{1,6}$, MICHAL BESSER ${ }^{5,6}$, RONI SHAPIRA ${ }^{2,6}$, ALON BEN-NUN $^{3}$, \\ DAVID GOITEIN $^{1}$, TIMA DAVIDSON ${ }^{4}$, ABRAHAM J. TREVES $^{5,6}$, GAL MARKEL $^{5,6}$, \\ JACOB SCHACHTER ${ }^{2,6}$ and MOSHE Z. PAPA ${ }^{1}$
}

\begin{abstract}
Departments of ${ }^{1}$ Surgical Oncology, ${ }^{2}$ Oncology, ${ }^{3}$ Thoracic Surgery, and ${ }^{4}$ Nuclear Medicine; ${ }^{5}$ Sheba Cancer Research Center;
${ }^{6}$ Ella Institute of Melanoma Research, Chaim Sheba Medical Center, Tel Hashomer, Israel
\end{abstract}

Received December 28, 2011; Accepted January 30, 2012

DOI: $10.3892 / \mathrm{etm} .2012 .498$

\begin{abstract}
Tumor-infiltrating lymphocytes (TILs) are produced by resecting tumor tissue and growing and expanding ex vivo large quantities of autologous $\mathrm{T}$ cells. Once the TILs are ready for infusion, the patient undergoes a non-myeloablative lympho-depleting course of chemotherapy and subsequent TIL infusion with high-dose bolus IL-2. This study reviews the surgical experience of the TIL program at the Chaim Sheba Cancer Research Center in Israel. Eligible patients underwent surgical consultation to determine what tumorectomy would be beneficial for harvesting appropriate tissue. Factors involved in the decision included tumor mass size, location and morbidity of the procedure. Between January 2006 and May 2010, 44 patients underwent 47 procedures of adoptive transfer of TILs. Three patients underwent the procedure twice for recurrence after initial good responses, including an additional surgical procedure to produce fresh tumor. Thirty-seven excisions were with general anesthesia and 10 were with local anesthesia. Of the 37 general anesthesia procedures, 27 were open procedures involving a thoracotomy, a laparotomy or dissection of a major lymph node basin. Ten used minimally invasive techniques such as thorascopy or laparoscopy. Tumorectomy sites included 18 lymph node metastasis, 13 subcutaneous nodules, 11 lung specimens and 5 abdominal visceral metastasis including 2 liver lesions. Surgical mortality and major morbidity was $0 \%$. Minor morbidity included only wound complications. Maximal number of TILs were derived from lymph node specimens, while liver metastasis procured the fewest TILs. Adoptive cell transfer technology affords a maximal tumor response with minimal surgical morbidity in metastatic patients.
\end{abstract}

Correspondence to: Dr Douglas Zippel, Department of Surgical Oncology, Chaim Sheba Medical Center, Tel Hashomer, Israel

E-mail: dougbz@gmail.com

Key words: adoptive cell therapy, melanoma, surgical oncology

\section{Introduction}

Metastatic melanoma has a poor prognosis, with a median survival of 6-10 months $(1,2)$. Treatment options for metastatic melanoma patients are very limited. Currently, interleukin-2 (IL-2) and dacarbazine (DTIC) remain the standard of care for metastatic melanoma, while other therapeutic options are still undergoing clinical trials (3). It is generally accepted that surgery has no role in the treatment of metastatic patients except in certain special circumstances.

Recently, new breakthroughs are being made in the technology of adoptive cell transfer using tumor-infiltrating lymphocytes (TILs) (4-7). TILs are produced by resecting tumor tissue and growing and expanding ex vivo large quantities of these autologous T cells. Once the TILs are ready for infusion, the patient undergoes a non-myeloablative lympho-depleting course of chemotherapy, and then receives the TIL infusion in conjunction with high-dose bolus IL-2 to maintain the activity of the cells in vivo. Using this technology, we previously reported a tumor response in up to $50 \%$ of refractory patients treated, as well as complete remissions in $13 \%$ of patients $(8,9)$. These results surpass the current accepted response rate of IL-2-based regimens of approximately $20 \%(10,11)$.

However, this protocol of treatment is a very labor-intensive procedure that requires considerable multi-disciplinary cooperation. In particular, given that all patients need fresh tumoral tissue to procure TILs, all patients must undergo a surgical procedure to harvest tumor for the laboratory. Since the population of metastatic melanoma patients is very heterogeneous as far as the location and extent of their disease, as well as their particular performance status, the range of procedures carried out to accomplish tumor harvest is quite varied. This study reviews the overall surgical experience of the TIL program of the Ella Institute of Melanoma at the Chaim Sheba Cancer Research Center in Israel. As this technology is a burgeoning and growing field, currently performed in only several cancer centers worldwide, we believe it would be beneficial to report our experience concerning specific surgical aspects of this program, for the benefit of any surgical oncology unit contemplating this type of treatment modality. 


\section{Materials and methods}

Data regarding all patients enrolled in the TIL program are kept in an ongoing prospective database. The records of all patients undergoing treatment in the TIL program were reviewed. Patients signed an informed consent approved by the Israeli Ministry of Health Approval No. 3518/2004 (ClinicalTrials.gov Identifier NCT00287131). Amendment for the use of 'young' short term cultured bulk TIL was approved in October 2007. Eligibility for the TIL program is stage IV melanoma, patient age over 18 years, negative for HIV, hepatitis B and C, and an Eastern Cooperative Oncology Group (ECOG) performance status of 0 or 1 with a life expectancy of at least 3 months. All patients had measurable disease by CT scan or physical diagnosis. Patients were enrolled after failure or relapse of IL-2-based therapies.

The TIL protocol has been previously extensively described by Dudley et al (12) and recently by Itzhaki et al (9). In brief, eligible patients underwent surgical consultation to determine what tumorectomy would be beneficial for harvesting appropriate tissue. Factors involved in the decision included tumor mass size, location, degree of dissemination and morbidity of the procedure. The minimum size of a resected tumor was at least 2 cubic centimeters in volume. The tumor was readily accessible for surgical extirpation. The mass was best chosen when heavily infiltrated by the melanoma tumor, usually discernable by strong PET avidity. Once the appropriate surgical procedure was decided upon, the patient underwent surgery in coordination with the TIL laboratory staff to assure timely acquisition of fresh tumor tissue. The excised tumor was transferred to a sterile container with saline and brought within a short time to the laboratory, usually within $1 \mathrm{~h}$ of excision. The tumor was sliced into small pieces, approximately 2-3 $\mathrm{mm}$ in size (13). The tumor fragments were then transferred to 24-well tissue culture plates for generating TIL cultures for future expansion. The first 12 treated patients in the program underwent the 'selected' TIL protocol, in which different TIL cultures were co-cultured with autologous melanoma tissue and evaluated for IFN- $\gamma$ release as described by Dudley et al (12). However, subsequent patients underwent a modification of the original protocol called 'young' TILs, in which the TIL cultures were grown as one bulk after pooling all lymphocytes together $(9,13,14)$. This modification has resulted in a shorter time to TIL infusion and more promising responses, as we previously reported $(8,9)$. Selected TILs are ready for infusion approximately 5-7 weeks after tissue procurement, while young TILs can be ready within 4-5 weeks of tissue procurement. In both techniques, after establishing a sufficient number of cells, between $30-50 \times 10^{6}$ cells, the TILs are rapidly expanded 1000 -fold to treatment levels, in a process called REP (rapid expansion), reaching approximately $40 \times 10^{9}$ cells . The medium is then drastically reduced by apheresis prior to administration (15).

Seven days prior to TIL infusion, non-myeloablative chemotherapy was administered to deplete the population of current lymphocytes in the patients. The regimen included cyclophosphamide $(60 \mathrm{mg} / \mathrm{kg})$ on days 7 and 6 and fludarabine $\left(25 \mathrm{mg} / \mathrm{m}^{2}\right)$ on days $5-1$. On day 0 , TILs were administered intravenously over $30 \mathrm{~min}$ followed by bolus high-dose IL-2 every $8 \mathrm{~h}$ to tolerance. Response to treatment was evaluated by CT scan 4 weeks after treatment and every 3 months subsequently.

\section{Results}

Patient demographics and treatment protocols. Between January 2006 and May 2010, 44 patients underwent adoptive transfer of TILs. Three patients underwent the procedure twice, for recurrence at 20,13 and 12 months after their initial procedures, resulting in a total of 47 treatments. These 3 patients had an additional surgical procedure to procure fresh tumor for their second infusion. The first 12 patients received 'selected' TIL infusions. Thirty-two patients underwent infusion with 'young' TILs. An additional 2 patients underwent both selected and young TIL infusion. One patient underwent young TIL infusion twice. One patient did not receive cyclophosphamide in the depletion chemotherapy regimen, due to being heavily pretreated in the past with chemotherapy. The cohort is described in Table I.

Type of anesthesia. Of the 47 procedures carried out in order to procure the TILs, 37 procedures were conducted under general anesthesia, while the remaining 10 procedures were performed under local anesthesia. The 10 local anesthesia procedures were all excisions of subcutaneous tumor nodules, easily palpated and resected in a day surgery setting, involving minimal patient discomfort and without need for overnight hospitalization.

General anesthesia procedures. The 37 procedures performed under general anesthesia were major procedures requiring in-house hospitalization and overnight stay. These procedures involved resection of tumors either in lymph node basins, parenchymal metastatic tissue or very large subcutaneous lesions. Twenty-seven of these operations were 'open' invasive procedures, meaning an open thoracotomy, laparotomy or dissection of a major lymph node basin. Ten patients underwent minimally invasive surgery, by use of a thoracoscope or laparoscope.

The thoracoscopic procedures were all VATS (videoassisted thoracoscopic surgery) resections of lung parenchyma with metastatic lesions. The two laparoscopic procedures were one laparoscopic hepatic resection and one laparoscopic splenectomy.

The 47 procedures included various sources of tumor. The largest group were patients having lymph node excisions from the major lymph node basins, either axillary or groin, of which there were 18. Thirteen patients had subcutaneous nodules excised, including one breast lumpectomy for a metastatic breast mass. There were 11 lung excisions. Abdominal non-lymphatic excisions included two small bowel resections, two liver resections and one splenectomy. A summary of the operations performed is presented in Table II.

TIL numbers infused. The average number of TILs infused to all patients was $39.4 \times 10^{9}$ cells. There was no statistical difference between the different sources of tumor tissue used, except liver metastasis, of which there were only 2 cases. The liver metastases produced a much smaller amount of infused TILs. 
Table I. Characteristics of the patient cohort.

\begin{tabular}{|c|c|c|c|c|}
\hline No. of patients & Age (years) & Gender & D-T time (years) & Tumorectomy site \\
\hline 1 & 36 & $\mathrm{~F}$ & 5.5 & $\mathrm{LN}$ \\
\hline 2 & 51 & M & 10.5 & $\mathrm{SC}$ \\
\hline 3 & 57 & M & 1.5 & Lung \\
\hline 4 & 55 & M & 3.5 & Lung \\
\hline 5 & 56 & M & 3.0 & $\mathrm{SC}$ \\
\hline 6 & 53 & M & 4.75 & $\mathrm{LN}$ \\
\hline 7 & 56 & M & 5.3 & Lung \\
\hline 8 & 66 & M & 3.4 & $\mathrm{LN}$ \\
\hline 9 & 33 & M & 2.0 & 1-LN 2-small bowel \\
\hline 10 & 54 & M & 1.75 & $\mathrm{LN}$ \\
\hline 11 & 53 & $\mathrm{~F}$ & 4.75 & $\mathrm{LN}$ \\
\hline 12 & 69 & M & 4. 0 & $\mathrm{SC}$ \\
\hline 13 & 49 & M & 13.0 & $\mathrm{LN}$ \\
\hline 14 & 49 & M & 27.0 & 1-Lung 2-SC \\
\hline 15 & 36 & M & 3.0 & $\mathrm{LN}$ \\
\hline 16 & 36 & M & 10.0 & 1-SC 2-LN \\
\hline 17 & 57 & M & 16.3 & $\mathrm{LN}$ \\
\hline 18 & 41 & M & 2.0 & $\mathrm{SC}$ \\
\hline 19 & 60 & M & 5.0 & Lung \\
\hline 20 & 22 & M & 1.8 & Lung \\
\hline 21 & 34 & $\mathrm{~F}$ & 2.75 & Liver \\
\hline 22 & 41 & $\mathrm{~F}$ & 5.5 & Breast \\
\hline 23 & 45 & M & 1.0 & $\mathrm{LN}$ \\
\hline 24 & 57 & M & 2.4 & $\mathrm{LN}$ \\
\hline 25 & 66 & M & 16.0 & Small bowel \\
\hline 26 & 61 & $\mathrm{M}$ & 4.0 & Lung \\
\hline 27 & 71 & M & 2.3 & $\mathrm{SC}$ \\
\hline 28 & 49 & $\mathrm{~F}$ & n.a. & $\mathrm{SC}$ \\
\hline 29 & 68 & $\mathrm{~F}$ & 22.0 & Lung \\
\hline 30 & 52 & M & 0.5 & Liver \\
\hline 31 & 58 & M & 3.3 & $\mathrm{SC}$ \\
\hline 32 & 41 & M & 2.75 & $\mathrm{SC}$ \\
\hline 33 & 70 & $\mathrm{~F}$ & 2.2 & $\mathrm{LN}$ \\
\hline 34 & 36 & $\mathrm{~F}$ & 7.0 & Lung \\
\hline 35 & 49 & M & 8.2 & Spleen \\
\hline 36 & 66 & M & n.a. & $\mathrm{SC}$ \\
\hline 37 & 29 & $\mathrm{~F}$ & 3.5 & $\mathrm{LN}$ \\
\hline 38 & 60 & $\mathrm{~F}$ & 2.3 & $\mathrm{SC}$ \\
\hline 39 & 57 & M & 5.9 & $\mathrm{LN}$ \\
\hline 40 & 48 & $\mathrm{M}$ & 5.3 & Lung \\
\hline 41 & 43 & $\mathrm{~F}$ & 0.5 & $\mathrm{LN}$ \\
\hline 42 & 52 & $\mathrm{M}$ & 4.0 & $\mathrm{LN}$ \\
\hline 43 & 63 & $\mathrm{M}$ & 6.0 & $\mathrm{LN}$ \\
\hline 44 & 55 & $\mathrm{M}$ & 2.75 & Lung \\
\hline Mean & 51.4 & $33 \mathrm{M} / 11 \mathrm{~F}$ & 5.7 & \\
\hline
\end{tabular}

D-T time, time from melanoma diagnosis to TIL infusion. LN, lymph node; SC, subcutaneous nodule; n.a., not applicable.

Clinical data. Mortality and major morbidity for all 47 surgical procedures was $0 \%$. Minor morbidity related to surgery comprised of only minor wound infections. In no cases was TIL infusion delayed due to a surgical complication. 
Table II. Summary of the surgical operations ${ }^{\mathrm{a}}$.

\begin{tabular}{|c|c|c|}
\hline \multicolumn{3}{|l|}{ Type of anesthesia } \\
\hline General & & 37 \\
\hline Local & & 10 \\
\hline \multicolumn{3}{|c|}{ Operations under general anesthesia } \\
\hline Minimally invasive & & 10 \\
\hline Abdomen & 2 & \\
\hline Lung & 8 & \\
\hline Open invasive & & 27 \\
\hline \multicolumn{3}{|l|}{ Source of tumor } \\
\hline Subcutaneous nodules & & 13 \\
\hline Breast & 1 & \\
\hline Lymph node basin & & 18 \\
\hline Thorax & & 11 \\
\hline Abdomen & & 5 \\
\hline Bowel resection & 2 & \\
\hline Liver resection & 2 & \\
\hline Splenectomy & 1 & \\
\hline \multicolumn{3}{|c|}{ Average number of Tils ( $\left.\times 10^{9}\right)$} \\
\hline Subcutaneous & & 36.19 \\
\hline Lymph node basin & & 45.16 \\
\hline Thorax & & 42.10 \\
\hline Small bowel/spleen & & 48.60 \\
\hline Liver & & 14.50 \\
\hline
\end{tabular}

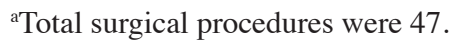

In a previous report, we published data on the clinical responses of 20 young-TIL patients (8). Out of 20 evaluated patients $10(50 \%)$ patients achieved an objective response rate according to the Response Evaluation Criteria In Solid Tumors (RECIST) guidelines (16), including two ongoing complete remissions and eight partial responses (PR). Presently, overall evaluable patients of the TIL program include $15(48 \%)$ of 31 evaluated patients experiencing an objective response (OR), among them 4 complete and 11 partial responses. Nine patients show disease stabilization (SD) with 2 having a minor response, although not qualifying them as partial responders. Seven patients progressed after treatment (PD).

\section{Discussion}

Adoptive cell therapy with TILs is proving to be an effective treatment for metastatic melanoma in patients who have failed current standard therapy $(8,17)$. However, due to its complex nature, with the many varied aspects which are involved in procuring and administering TILs, this technology requires a considerable multi-disciplinary infrastructure. The labor intensive nature of TIL protocols has been a limiting factor in its widespread use. Our program has increasingly looked for ways to streamline the process in order to both simplify the technique and increase the efficacy of the infused lymphocytes. The surgical oncology team is a critical part of the overall effort and must work in close coordination with the laboratory and the medical oncology team.
It is of vital importance that the fresh tissue be made readily available to the laboratory within a short time of surgical extirpation. In general, our specimens were often made available to the laboratory within minutes of surgery, before the patient had left the operating room. The tissue was transported to the laboratory in sterile containers and on the cutting board and ready for fragmentation or digestion within one hour. This was to ensure that a maximal number of TILs would be available and viable for starting the tissue cultures.

This may have significant clinical impact. We have already shown that infusing a larger amount of TILs into a patient was positively correlated with response to treatment (8). This is probably due to the high concentration of CD8 killer T cells in the infusion. Interestingly, our 2 liver resections produced the fewest number of TILs. The TIL program of the Surgical Branch of the National Institutes of Health (NIH) recently reported their experience with hepatic resections for TIL procurement (18). In their series, 13 patients underwent TIL infusion derived from hepatic lesions with an overall response rate of $40 \%$ (6 patients). The NIH group also reported that it was more difficult to grow TILs from gastrointestinal metastasis, excluding the liver, than from lung, lymph node or liver metastases. The small number of liver tumor resections precludes drawing definitive conclusions from our data. However, we were able to grow a sufficiently large amount of TILs for therapy from 2 gastrointestinal resections and 1 spleen metastasis, while liver tissue yielded much fewer cells. In general, the laboratory has successfully been able to grow TILs from almost any tissue source, so that the surgical procedure is by most part decided by tumor mass size and surgical accessibility and morbidity of the procedure.

Another vital aspect of the program is to have the patient ready in time for treatment. Since switching to the young TIL protocol, the goal of the laboratory team is to have a sufficient number of TILs ready for infusion by 28 days after starting tissue culture. The non-myeloablative chemotherapy regimen begins 7 days before TIL infusion. Thus, the patient must be fit and ready to undergo treatment at 3 weeks post surgery. For minor procedures carried out under local anesthesia this is generally not a problem. But when more complex procedures are conducted, particularly involving metastasectomy from the thorax or abdomen, surgical complication can needlessly delay treatment. Therefore, the choice of which procedure to conduct to procure the tumor may affect the timing of subsequent treatment. Because of this limitation, it is always in our interest to attempt thoracic or abdominal resections in a minimally invasive manner where possible. This is in order to keep morbidity to a minimum as not to delay treatment because of surgical complications. In our cohort of 47 procedures, 37 of which were major operations under general anesthesia, we did not experience any mortality or morbidity which precluded treatment.

When we used the selected TIL protocol which involved a longer process to produce treatment-ready TILs, one of the concerns was patient drop out due to disease progression during the wait until the cells were ready for infusion (13). The use of young TILs, as well as safe low-morbid surgery has contributed to almost all enrolled patients succeeding in undergoing the treatment regime without delay.

It is of note that this platform of adoptive cell transfer with TILs can be adapted for use in other solid tumors. A Swedish 
research group recently published a study using adoptive cell transfer with TILs procured from sentinel lymph nodes in the treatment of advanced colorectal cancer with promising results (19). Once the infrastructure of the laboratory process of producing TILs is in place, the use of other tumoral tissue does not require any major changes to the protocol. Thus, the use of this technology can be readily expanded to include many other tumor types.

The TIL program is an ongoing program which we are learning to improve as we go along, and it is our hope that as we gain more and more experience, we can improve the quality of care for these high-priority patients. In conclusion, this study examined the specific surgical aspects of adoptive cell transfer with TILs for metastatic melanoma. Our experience has shown that careful surgical planning in conjunction with a high degree of multi-disciplinary cooperation allows for successful implementation of such a program within a large university-based hospital setting. In addition, this technology affords a maximal tumor response with minimal surgical morbidity for metastatic melanoma patients.

\section{References}

1. Khan MA, Andrews S, Ismail-Khan R, et al: Overall and progression-free survival in metastatic melanoma: analysis of a single-institution database. Cancer Control 13: 211-217, 2006.

2. Tarhini AA and Agarwala SS: Cutaneous melanoma: available therapy for metastatic disease. Dermatol Ther 19: 19-25, 2006.

3. Tsao H, Atkins MB and Sober AJ: Management of cutaneous melanoma. N Engl J Med 351: 998-1012, 2004.

4. Rosenberg SA and Dudley ME: Adoptive cell therapy for the treatment of patients with metastatic melanoma. Curr Opin Immunol 21: 233-240, 2009.

5. Rosenberg SA, Restifo NP, Yang JC, et al: Adoptive cell transfer: a clinical path to effective cancer immunotherapy. Nat Rev Cancer 8: 299-308, 2008.

6. Dudley ME and Rosenberg SA: Adoptive cell transfer therapy. Semin Oncol 34: 524-531, 2007.

7. Dudley ME, Yang JC, Sherry R, et al: Adoptive cell therapy for patients with metastatic melanoma: evaluation of intensive myeloablative chemoradiation preparative regimens. J Clin Oncol 26: 5233-5239, 2008.
8. Besser MJ, Shapira-Frommer R, Treves AJ, et al: Clinical responses in a phase II study using adoptive transfer of short-term cultured tumor infiltration lymphocytes in metastatic melanoma patients. Clin Cancer Res 16: 2646-2655, 2010.

9. Itzhaki O, Hovav E, Ziporen Y, et al: Establishment and large scale expansion of minimally cultured 'young' tumor infiltrating lymphocytes for adoptive transfer therapy. J Immunother 34: 212-220, 2011.

10. Dutcher J: Current status of interleukin-2 therapy for metastatic renal cell carcinoma and metastatic melanoma. Oncology (Williston Park) 16: 4-10, 2002.

11. Atkins MB, Hsu J, Lee S, et al: Phase III trial comparing concurrent biochemotherapy with cisplatin, vinblastine, dacarbazine, interleukin-2, and interferon alfa-2b with cisplatin, vinblastine, and dacarbazine alone in patients with metastatic malignant melanoma (E3695): a trial coordinated by the Eastern Cooperative Oncology Group. J Clin Oncol 26: 5748-5754, 2008.

12. Dudley ME, Wunderlich JR, Shelton TE, et al: Generation of tumor-infiltrating lymphocyte cultures for use in adoptive transfer therapy for melanoma patients. J Immunother 26: 332-342, 2003.

13. Besser MJ, Shapira-Frommer R, Treves AJ, et al: Minimally cultured or selected autologous tumor-infiltrating lymphocytes after a lympho-depleting chemotherapy regimen in metastatic melanoma patients. J Immunother 32: 415-423, 2009.

14. Tran KQ, Zhou J, Durflinger KH, et al: Minimally cultured tumor-infiltrating lymphocytes display optimal characteristics for adoptive cell therapy. J Immunother 31: 742-751, 2008.

15. Schallmach E, Sareli R, Besser MJ, et al: Collection of largescale expanded lymphocyte cultures for adoptive immunotherapy using a COBE Spectra apheresis machine. J Immunother 31: 563-568, 2008.

16. Eisenhauer EA, Therasse P, Bogaerts J, et al: New response evaluation criteria in solid tumours: revised RECIST guideline (version 1.1). Eur J Cancer 45: 228-247, 2009.

17. Dudley ME, Wunderlich JR, Yang JC, et al: Adoptive cell transfer therapy following non-myeloablative but lymphodepleting chemotherapy for the treatment of patients with refractory metastatic melanoma. J Clin Oncol 23: 2346-2357, 2005.

18. Ripley RT, Davis JL, Klapper JA, et al: Liver resection for metastatic melanoma with postoperative tumor-infiltrating lymphocyte therapy. Ann Surg Oncol 17: 163-170, 2010.

19. Karlsson M, Marits P, Dahl K, et al: Pilot study of sentinel-nodebased adoptive immunotherapy in advanced colorectal cancer. Ann Surg Oncol 17: 1747-1757, 2010. 